\title{
VARIATION IN THE EFFECT OF ACETYLCHOLINE ON MYOMETRIAL ACTIVITY AND MATERNAL PLACENTAL BLOOD FLOW IN THE RABBIT
}

\author{
A. M. CARTER AND T. OLIN \\ Institute of Zoophysiology, University of Lund, and \\ Department of Diagnostic Radiology, University Hospital, Lund, Sweden
}

(Received 8th September 1972)

\begin{abstract}
Summary. The effect of acetylcholine upon uteroplacental blood flow was studied by $\mathrm{X}$-ray angiography following selective catheterization of the urogenital artery of the rabbit. Injection of acetylcholine into the artery usually increased the total uterine blood flow. In some cases, this entailed an increase in maternal placental blood flow but, in others, the blood supply to the placenta was cut off and the increased flow passed through the superficial myometrial vessels and, possibly, arteriovenous short circuits. The dichotomy in vascular response depended upon a variation in the myometrial response. In some animals, acetylcholine evoked a large contraction, which curtailed placental flow by compressing blood vessels. In others, the myometrium reacted slightly or not at all to acetylcholine and placental blood flow was able to increase. The vascular and myometrial responses to acetylcholine could be inhibited by previous administration of atropine or butylscopolamine.
\end{abstract}

\section{INTRODUCTION}

There is a widespread assumption that all components of the uteroplacental vascular bed react to stimuli in a similar way. We first questioned the validity of this assumption when $\mathrm{X}$-ray angiographic studies in the rabbit showed that a vasoconstrictor, vasopressin, reduced blood flow to the placenta more severely than to the uterine wall (Carter, Göthlin \& Bengtsson, 1968). Subsequently, Duncan (1969) provided convincing evidence that, during maternal hypoxaemia, maternal placental blood flow is severely reduced whilst myometrial blood flow is virtually unchanged. Both hypoxaemia and vasopressin caused a redistribution of uterine blood flow coincident with a reduction in total flow. We here report that maternal placental blood flow can be reduced even by a stimulus that increases total uterine blood flow.

\section{MATERIAL AND METHODS}

Twenty rabbits were used for this study, of which three were non-pregnant parous animals and the remainder were at known stages of pregnancy from 
15 to 31 days post coitum (full term occurs at 31 to 32 days). General anaesthesia was obtained with intravenous pentobarbitone sodium (Mebumalnatrium $6 \%, \mathrm{ACO}$, Sweden) and maintained at a constant level throughout the experiment. A radio-opaque catheter of polyethylene (OPP 60, Portex, England, o.d./i.d. $=1.22 \mathrm{~mm} / 0.76 \mathrm{~mm}$ ) with a bent tip portion was used for angiography. It was introduced into the femoral artery and the tapered tip was positioned in the mouth of the urogenital artery during fluoroscopy. Serial angiography was made with twofold or fourfold magnification using an automatic film changer with high definition scıeens (Carter, Göthlin \& Olin,

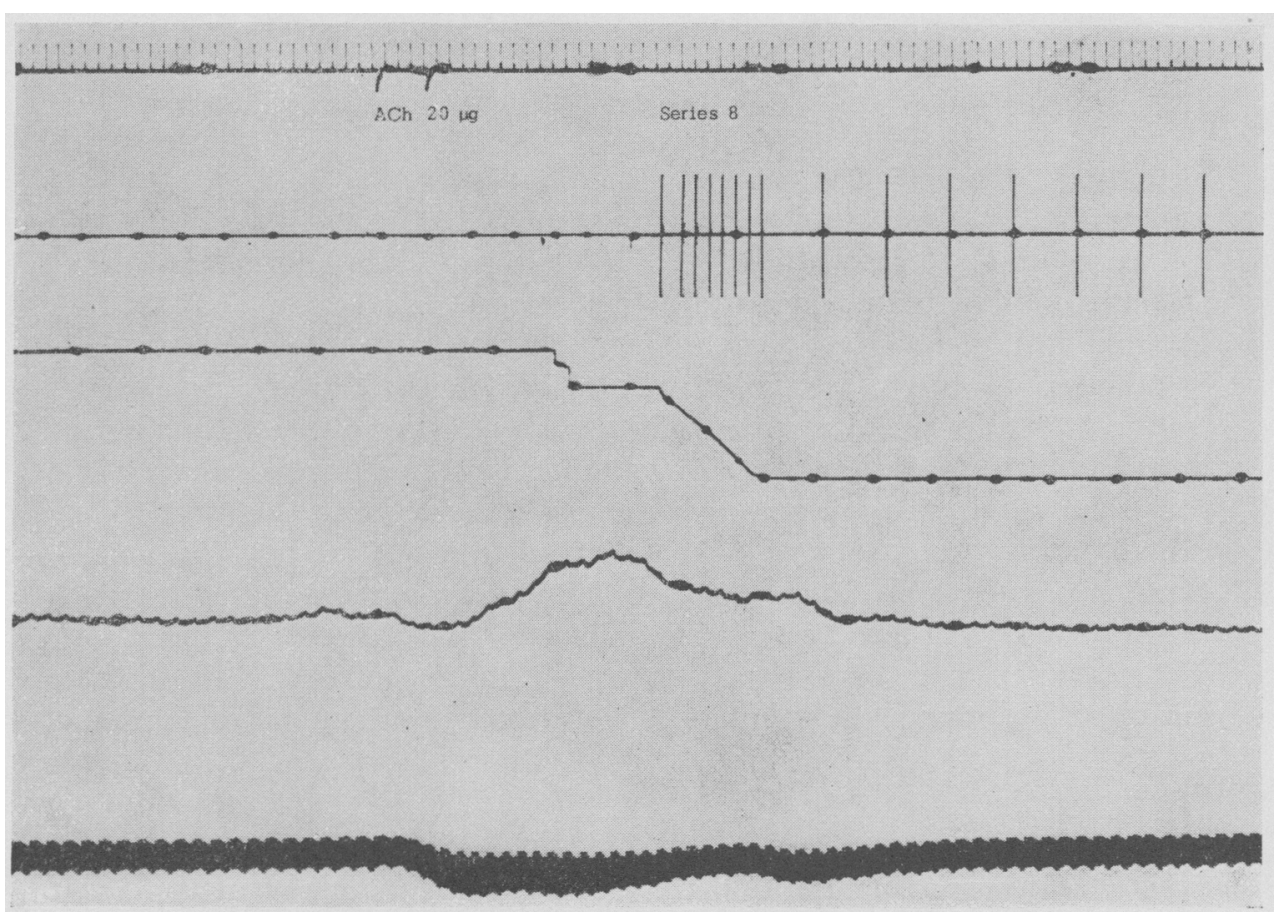

TEXT-FIG. 1. Polygraph record illustrating the experimental variables at pharmacoangiography in the rabbit. Records from above downwards: time in sec (upward deflections) and acetylcholine injection (downward deflections), film exposures, contrast medium injection, intrauterine pressure, and arterial blood pressure. The rate of contrast-medium injection is given by the slope of the corresponding curve, and inspection of the films shows whether it is greater, lesser or equal to the rate of blood flow. Features on the films can be related to the time course of contrast medium injection and to the intrauterine and arterial blood pressures, as well as to the time of acetylcholine administration. Note the rapid, yet transient fall in blood pressure after acetylcholine injection.

1971). Control series of angiograms were compared with series exposed at various intervals after injection of drugs through the angiography catheter.

The water-soluble contrast medium (meglumine metrizoate, Isopaque Cerebral, Nyco A/S, Norway) was injected with a high pressure syringe. The course of the injections was registered on a direct writing polygraph so that the exact rate of injection could be determined (Text-fig. 1). The polygraph also recorded the film exposures and the arterial blood pressure, measured electro- 
manometrically from a catheter in one of the common carotid arteries. The timing of the films could thereby be related to the contrast medium injection and the blood pressure changes.

Intrauterine pressure was recorded through a sponge-tipped, open-ended vinyl catheter (VX 010, Becton Dickinson, U.S.A., o.d./i.d. $=0.9 \mathrm{~mm} /$ $0.5 \mathrm{~mm}$ ), filled with isotonic saline (Carter, Naaktgeboren \& Van Zon-Van

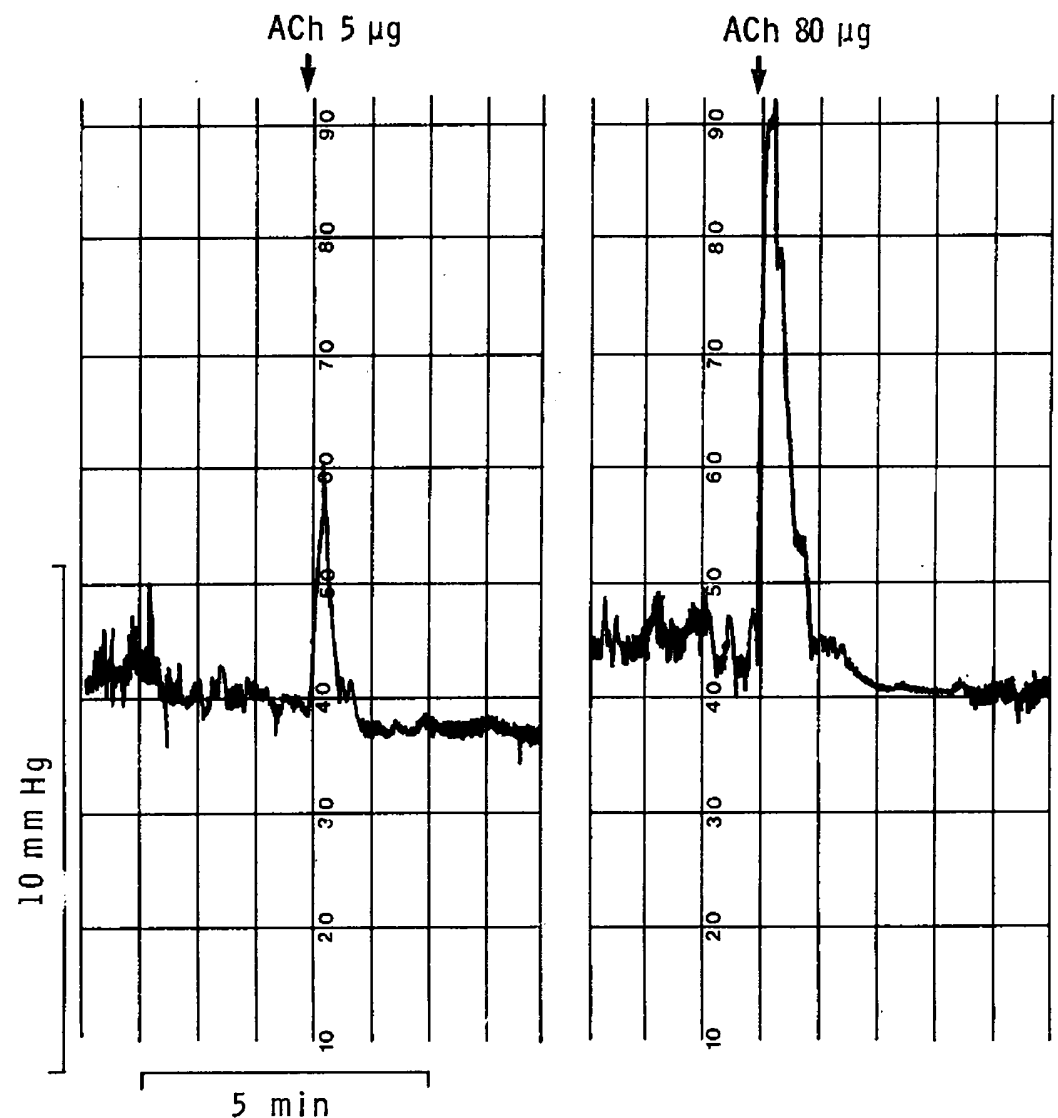

TEXT-FIG. 2. Intraluminal pressure recorded from the right uterine horn of a pregnant rabbit, 24 days post coitum. Selective injection of $5 \mu \mathrm{g}$ acetylcholine in the right urogenital artery caused a moderate contraction, amplitude about $5 \mathrm{~mm} \mathbf{H g}$, duration about $25 \mathrm{sec}$, followed by a period of inactivity. A dose of $80 \mu \mathrm{g}$ acetylcholine evoked a somewhat larger response, amplitude about $11 \mathrm{~mm} \mathrm{Hg}$, duration about $50 \mathrm{sec}$. Corresponding angiograms are shown in Pl. 2, Figs 2 and 3.

Wagtendonk, 1971). The catheter was placed between the endometrium and fetal membranes in the uterine horn on the same side as the angiography catheter, with its tip opposite one of the placentae. The pressure was measured electromanometrically and registered on a potentiometer writer together with film exposures (Text-fig. 2). Supplementary drug injections without angiography were made to define myometrial responses.

The drugs administered were acetylcholine chloride $(1 \mu \mathrm{g}$ to $1000 \mu \mathrm{g}$; Hoffmann-La Roche, Switzerland), atropine sulphate (1.25 $\mu \mathrm{g}$ to $2000 \mu \mathrm{g}$; 
ACO, Sweden) and butylscopolamine bromide (500 $\mu \mathrm{g}$ to $5000 \mu \mathrm{g}$; Buscopan ${ }^{\star}$, Boehringer, Ingelheim, West Germany).

\section{RESULTS}

The myometrial response to acetylcholine was a single contraction, followed by a period of relative quiescence with reduced tonus. The size of the response increased only gradually with the dose and there was considerable variation from one animal to another. In some rabbits, the intrauterine pressure increase exceeded $10 \mathrm{~mm} \mathrm{Hg}$. The stage of gestation seemed to affect the response, which was most pronounced before the 24th day of gestation and towards term.

The blood flow in the urogenital artery was estimated from the known rate of injection of contrast medium and the extent to which the medium was diluted in or spilled over from, this vessel (Olin \& Redman, 1966; Carter, Göthlin \& Olin, 1971). The distribution of total uterine blood flow between the uterine wall and the placentae could be assessed at the end of contrast-medium injection by evaluating the opacity of the myometrial and placental vessels, as this is dependent upon the amount of contrast reaching them. A first analysis showed a marked variation in the response of the uteroplacental vascular bed to acetylcholine. There was generally an increase in uterine blood flow and dilatation of the uterine artery and its major branches. In many animals, however, little or none of the injected contrast medium entered the placental vessels (P1. 1, Fig. 4). In these rabbits, the larger myometrial vessels were well filled with contrast medium but finer vessels were less well seen. Contrast medium entered the uterine veins earlier and in higher concentration than in the control series (Pl. 1, Fig. 4). In a second group of animals, the contrast medium entered the placentae as usual and it reached the periphery of the placentae faster (Pl. 2, Fig. 6). The veins were seen earlier than in the control series and showed a greater opacity, although the difference was a moderate one.

Most rabbits could be assigned to one or other of the above groups, there being few intermediates. In some animals, however, there were variations in the effect of even the same dose of acetylcholine. These included experiments in which we deliberately varied the delay between the acetylcholine injection and angiography so that the latter would fall before, during or after the myometrial contraction. A decrease in maternal placental blood flow was seen only when angiography was performed during the contraction. A re-examination of the rest of the material confirmed that the variation in the effect of acetylcholine on the placental blood flow was directly dependent upon variations in the myometrial response (Text-fig. 2; Pl. 2, Figs 6 and 7).

Although the acetylcholine was given selectively in the urogenital artery, a decrease in blood pressure occurred after a rather short interval (Text-fig. 1). If the dose was too high, i.e. $100 \mu \mathrm{g}$, a large fall in perfusion pressure led to decreased flow in the urogenital artery and a dimunition of vessel calibre.

After previous injection of atropine or butylscopolamine, acetylcholine had no effect upon the uterine blood vessels or upon uterine blood flow. The same 
PLATE 1
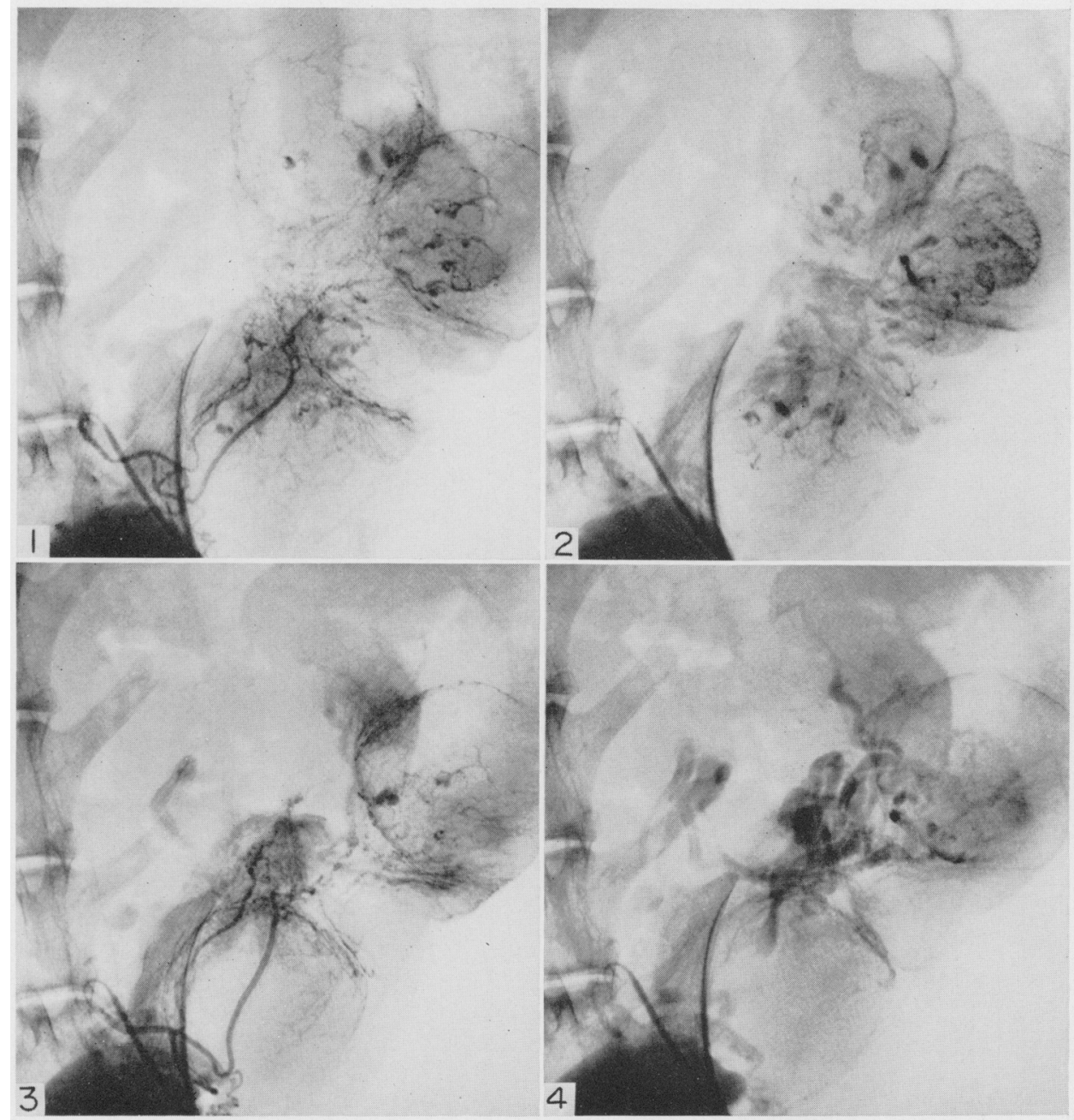

Fig. 1. Angiography of the left urogenital artery in a pregnant rabbit, 23 days post coitum. Late arterial phase. The rate of contrast-medium injection $(0 \cdot 15 \mathrm{ml} / \mathrm{sec})$ is in equilibrium with the rate of blood flow. There is contrast medium in the myometrial arteries and in the placental arterial sinuses.

Fig. 2. Same as Fig. 1, but $10 \mathrm{sec}$ later. Contrast medium pools in the placental sinuses. There is only slight opacity of the uterine veins.

FIG. 3. Repeat angiography, after $10 \mu \mathrm{g}$ acetylcholine (intrauterine pressure peak about $8 \mathrm{~mm} \mathrm{Hg}$ ). Late arterial phase. The rate of blood flow now exceeds the rate of contrastmedium injection $(0.18 \mathrm{ml} / \mathrm{sec})$ as evidenced by admixture of blood in the contrast stream. The opacity of the placental vessels is diminished, but the myometrial vessels are well filled. Contrast medium is already discernible in the uterine veins.

Fig. 4. Same as Fig. 3, but $10 \mathrm{sec}$ later. There is hardly any contrast medium in the placental sinuses. The opacity of the uterine veins is strongly increased, secondary to an increase in myometrial blood flow.

(Facing p. 76) 
PLATE 2
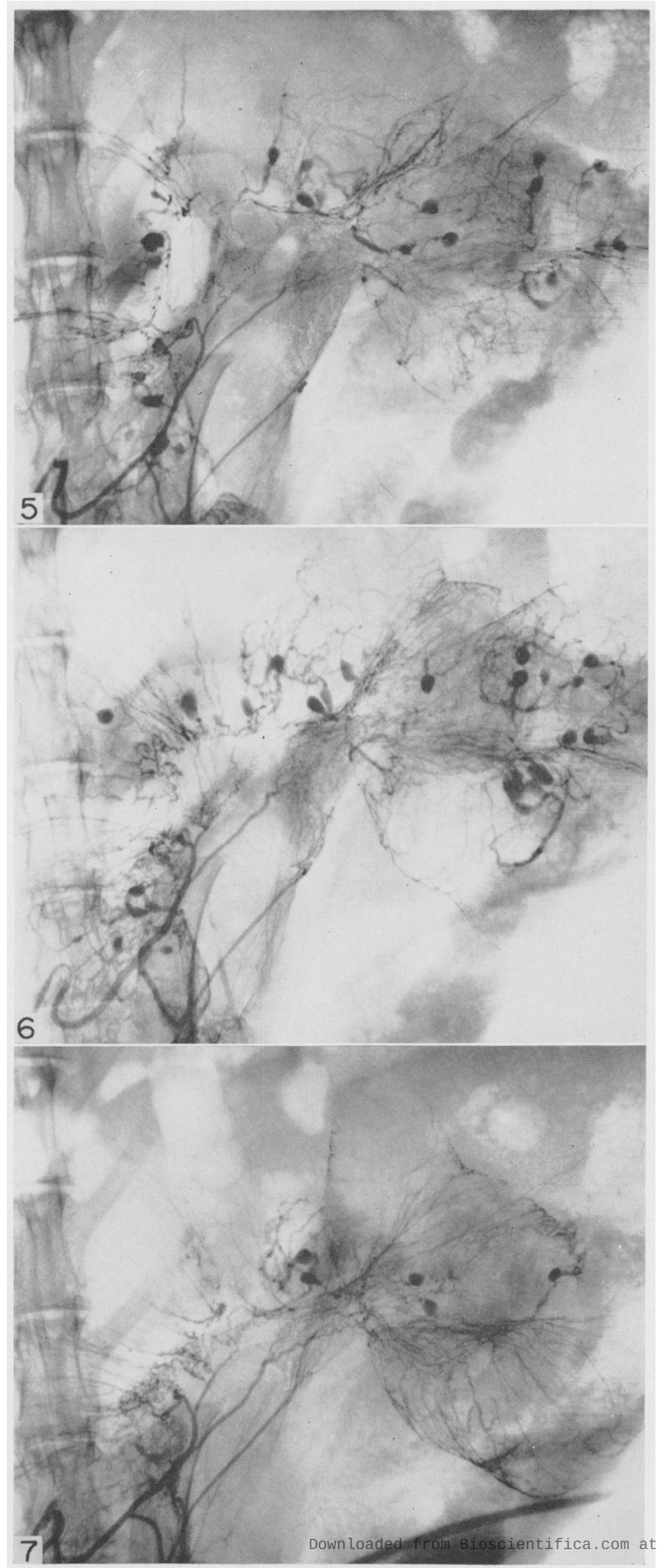
drugs also inhibited acetylcholine-induced myometrial contraction and the circulatory effects attributable to it.

Atropine alone usually, but not consistently, caused constriction of the uterine arteries and their branches, but did not markedly affect uteroplacental circulation. Vasoconstriction was sometimes seen when large doses of atropine were given together with acetylcholine. Atropine alone had no marked effect upon the intrauterine pressure pattern.

\section{DISGUSSION}

The most striking finding of the present study is the remarkable redistribution of uterine blood flow which can be caused by acetylcholine and the dependence of this effect upon the myometrial response.

An increase in volume flow through the urogenital artery was a consistent response to acetylcholine. This effect was not unexpected, as acetylcholine is among the few substances that have been shown to improve uterine blood flow in pregnant animals (Ahlquist, 1950; Greiss, Gobble, Anderson \& McGuirt, 1967b). Earlier filling of the veins with contrast medium was also a common feature, suggesting a decrease in circulation time.

Where there was no impairment of placental filling, it seemed that the rate of placental blood flow had increased in proportion to the increase in total uterine flow. The contrast medium traversed the placentae faster, however, and it is therefore doubtful whether the increase in blood flow benefited placental exchange. In many placentae, including those of the rabbit, rat, sheep, rhesus monkey and man, there are elaborate vascular arrangements for reducing the velocity of blood flow, which strongly suggests that a slow, even perfusion is most beneficial to placental exchange (Carter, 1973).

A strong reduction in placental filling was generally associated with a marked increase in intrauterine pressure. The resistance to flow in a vessel is not only influenced by vasomotion: it can be increased due to compression by surrounding tissues (Folkow \& Neil, 1971). It is well known that labour contractions cause a temporary impairment of maternal placental blood flow (Ramsey, Corner \& Donner, 1963; Borell, Fernström, Ohlson \& Wiqvist, 1965). In the rabbits studied, spontaneous activity rarely caused intrauterine pressure peaks

\section{EXPLANATION OF PLATE 2}

FIG. 5. Angiography of the right urogenital artery in a pregnant rabbit, 24 days post coitum (the right horn has shifted to the left side). Late arterial phase. The injection rate $(0.24 \mathrm{ml} / \mathrm{sec})$ exceeds the blood flow rate: there is spillover of contrast medium to the internal iliac artery. The appearance of the myometrial vessels and placental sinuses is normal.

FIG. 6. Repeat angiography after $5 \mu$ g acetylcholine, which caused a moderate myometrial contraction with its peak before the start of contrast medium injection (cf. Text-fig. 2). The rate of blood flow is now greater than the injection rate $(0.25 \mathrm{ml} / \mathrm{sec})$ : there is admixture of blood and contrast medium in the urogenital artery. The uterine artery has widened by about $40 \%$. Filling of the myometrial vessels and placental sinuses with contrast medium has increased.

Fic. 7. Repeat angiography after $80 \mu \mathrm{g}$ acetylcholine. There was a strong myometrial contraction during the arterial phase (cf. Text-fig. 2). The myometrial vessels are well filled but there is poorer filling of the placental sinuses than in earlier series. 
in excess of $5 \mathrm{~mm} \mathrm{Hg}$, whereas the response to acetylcholine frequently exceeded $10 \mathrm{~mm} \mathrm{Hg}$. Faced with a massive increase in resistance to flow through the placentae, the contrast medium apparently took an alternative route. A great deal seems to have passed to the major vessels in the uterine wall, which were clearly seen (cf. Pl. 2, Fig. 7), and it also appeared rapidly in the uterine veins. The high density of contrast medium in the veins can mainly be ascribed to the impaired placental circulation. Usually, contrast medium leaving the myometrium is diluted by blood leaving the placentae, as the placental circulation time is considerably longer than the myometrial circulation time (Carter, Göthlin \& Olin, 1971).

The morphological prerequisites for a redistribution of blood flow from the placentae to the uterine wall exist, as the arteries supplying the two areas anastomose freely (Carter, Göthlin \& Olin, 1971). It may not be immediately apparent, however, why resistance to flow increases more in the non-contractile placentae than in the contracting uterine wall. Two factors must be borne in mind: firstly, there must be a pressure gradient from the uterine lumen to the serosa, comparable to that in a strongly contracting artery (Burton, 1962); secondly, the larger vessels in the uterine wall are rather superficial (Chipman, 1902), and therefore at the lower end of the gradient, whereas the placentae and our recording catheter were in the uterine lumen. The smaller vessels feeding the inner portion of the myometrium and the endometrium may well be closed when the uterus contracts so that the increase in blood flow to the uterine wall should not be equated with an increase in nutritive flow. A parallel may be drawn with the distended colon, in which serosal flow persists but mucosal flow is impaired (Dencker, Lingårdh, Muth \& Olin, 1969).

In addition to a redirection of blood through the uterine wall, opening of arteriovenous short circuits may enable blood to bypass the uterus completely. This would also lead to earlier appearance of undiluted contrast medium in the veins. There is fairly strong evidence that short circuits exist (Carter, Göthlin \& Olin, 1971) and they may well be opened by acetylcholine. The uterine venous system is extremely extensive in the rabbit, accounting for about one-sixth of the total blood volume (Barcroft \& Rothschild, 1932). If these vessels dilate to even a minor extent, there should be a considerable reduction in venous return. This might explain the prompt fall in blood pressure which occurred at selective injection of acetylcholine in the urogenital artery.

Some X-ray contrast media inhibit a variety of enzymes, including acetylcholinesterase (Lasser \& Lang, 1966). The degree of inhibition is least for the less hydrophobic compounds such as diatrizoate (Lang \& Lasser, 1971). The contrast medium used in this study was metrizoate, which closely resembles the latter substance in its molecular structure, and therefore is unlikely to have potentiated the action of endogenous or exogenous acetylcholine.

The uterine vasculature and myometrium both exhibited responses to acetylcholine that could be inhibited by atropine or butylscopolamine. It does not, however, follow that they receive a cholinergic innervation. Parasympathetic nerve stimulation elicits a myometrial contraction not unlike that evoked by parasympathomimetics, but this response is resistant to blockade by atropine (Cushny, 1906). A similar atropine resistance occurs in the bladder and recent 
studies of this organ suggest that most of the receptors stimulated by acetylcholine play no part in parasympathetic nervous transmission, which seems to be mediated by some other substance (Ambache \& Zar, 1970; Dumsday, 1971). It is a reasonable assumption that a similar situation exists in the myometrium.

There is convincing evidence that the uterine vasculature of the sheep lacks cholinergic innervation, either sympathetic or parasympathetic, although it dilates in response to acetylcholine and is constricted by atropine (Greiss \& Gobble, 1967; Greiss, Gobble, Anderson \& McGuirt, 1967a, b). Histochemical observations are in accordance with these findings. Although nerves staining for acetylcholinesterase are associated with the main uterine arteries of several species, they are absent in the sheep, as well as in the cat, rat, cow and rabbit (Bell, 1971). Thus, the uterine vascular response to acetylcholine in the rabbit must depend upon the stimulation of receptors unassociated with nervous transmission.

Constriction of the uterine arteries of the guinea-pig in response to atropine has been cited as evidence for an active cholinergic dilatation of these vessels during pregnancy (Bell \& Brown, 1971). Since we have obtained a similar result in the rabbit, by a similar technique, the validity of this evidence seems open to question. It was suggested by Greiss et al. (1967a) that the uterine vasoconstriction evoked by atropine in sheep is due to a general imbalance of the autonomic nervous system, with sympathetic overactivity. A similar explanation could be offered for the rabbit and cannot be precluded in the guinea-pig.

The idea that uterine contractions may affect placental blood flow is by no means new (Robson \& Schild, 1938). Hitherto, however, most evidence for this view has been provided by experiments where total uterine blood flow was reduced. We are not aware of any previous report of a reduction in placental blood flow accompanied by an increase in total uterine blood flow. The only techniques that can reveal information of this type are angiography and, possibly, isotope-labelled microsphere methods. It may thus be difficult to demonstrate whether acetylcholine has a detrimental effect on maternal placental circulation in man. It is, however, established that prolonged infusion of acetylcholine can increase the frequency and amplitude of uterine contractions in pregnant women (Sala \& Fisch, 1965).

\section{ACKNOWLEDGMENTS}

This investigation was supported by the Swedish Medical Research Council (Project No. B72-23X-605-06A) and a grant from the Prenatal Research Fund.

\section{REFERENCES}

AHLQUIST, R. P. (1950) The action of various drugs on the arterial blood flow of the pregnant, canine uterus. F. Am. pharm. Ass. Scientific edn. 39, 370.

Ambache, N. \& ZAR, M. A. (1970) Non-cholinergic transmission by post-ganglionic motor neurones in the mammalian bladder. F. Physiol., Lond. 210, 761.

Barcroft, J. \& Rothschild, P. (1932) The volume of blood in the uterus during pregnancy. $\mathcal{F}$. Physiol., Lond. 76, 447. 
BELL, C. (1971) Distribution of cholinergic vasomotor nerves to the parametrial arteries of some laboratory and domestic animals. F. Reprod. Fert. 27, 53.

BeLL, C. \& BRowN, M. J. (1971) Arteriographic evidence for a cholinergic dilator mechanism in uterine hyperaemia of pregnancy in the guinea-pig. F. Reprod. Fert. 27, 59.

Borell, U., Fernström, I., OhLson, L. \& WiQvist, N. (1965) Influence of uterine contractions on the uteroplacental blood flow at term. Am. F. Obstet. Gynec. 93, 44.

Burron, A. C. (1962) Physical principles of circulatory phenomena: the physical equilibria of the heart and blood vessels. In: Handbook of Circulation. Vol. 1, p. 85. Eds. W. F. Hamilton and P. Dow. American Physiological Society, Washington, D.C.

Garter, A. M. (1973) Placental circulation. In: Introduction to the Placenta. Ed. D. Steven. Academic Press, London (in press).

Garter, A. M., Göthlin, J. \& Bengtsson, L. P. (1968) Effect of vasopressin on the uteroplacental circulation-an angiographic study in the rabbit. 7. Reprod. Fert. 17, 419.

Carter, A. M., Göthlin, J. \& Olin, T. (1971) An angiographic study of the structure and function of the uterine and maternal placental vasculature in the rabbit. F. Reprod. Fert. 25, 201.

Carter, A. M., NaAktgeboren, G. \& Van Zon-Van Wagtendonk, A. M. (1971) Parturition in the rabbit-Spontaneous uterine activity during pregnancy, parturition and the post partum period and its relation to normal behaviour. Eur. F. Obstet. Gynec. $1,37$.

Chipman, W. (1902) Observations on the placenta of the rabbit with special reference to the presence of glycogen, fat and iron. Rep. Lab. R. Coll. Physns, Edinb. 8, 225.

Cushny, A. R. (1906) On the movements of the uterus. F. Physiol., Lond. 35, 1.

Dencker, H., Lingårdh, G., MUTh, T. \& Olin, T. (1969) Massive gangrene of the colon secondary to carcinoma of the rectum. Acta chir. scand. 135, 357.

Dumsday, B. (1971) Atropine-resistance of the urinary bladder innervation. F. Pharm. Pharmac. 23, 222.

Duncan, S. L. B. (1969) The partition of uterine blood flow in the pregnant rabbit. F. Physiol., Lond. $204,421$.

Folkow, B. \& NeIL, E. (1971) Circulation. Oxford University Press, London.

Greiss, F. C. \& GobBLE, F. L. (1967) Effect of sympathetic nerve stimulation on the uterine vascular bed. Am. F. Obstet. Gynec. 97, 962.

Greiss, F. C., Gobble, F. L., Anderson, S. G. \& McGuirt, W. F. (1967a) Effect of parasympathetic nerve stimulation on the uterine vascular bed. Am. 7. Obstet. Gynec. 99, 1067.

Greiss, F. C., Gobrle, F. L., Anderson, S. G. \& MaGuirT, W. F. (1967b) Effect of acetylcholine on the uterine vascular bed. Am. 7. Obstet. Gynec. 99, 1073.

LANG, J. H. \& Lasser, E. G. (1971) Nonspecific inhibition of enzymes by organic contrast media. Jnl medicinal Chem. 14, 233.

LASSER, E. C. \& LANG, J. H. (1966) Inhibition of acetylcholinesterase by some organic contrast media. Investve Radiol. 1, 237.

Olin, T. \& Redman, H. (1966) Spillover flowmeter. A preliminary report. Acta radiol. (Diag.) 4, 217.

RAMSEY, E. M., CoRNER, G. W. \& DonNER, M. W. (1963) Serial and cineradioangiographic visualization of maternal circulation in the primate (hemochorial) placenta. Am. F. Obstet. Gynec. 86, 213.

Robson, J. M. \& Schild, H. O. (1938) Effect of drugs on the blood flow and activity of the uterus. 7. Physiol., Lond. 92, 9.

SALA, N. L. \& Fisch, L. (1965) Effect of acetylcholine and atropine upon uterine contractility in pregnant women. Am. 7. Obstet. Gynec. 91, 1069. 